ACCEPTED MANUSCRIPT

\title{
Rogue bioelectrical waves in the brain: the Hurst exponent as a potential measure for presurgical mapping in epilepsy
}

To cite this article before publication: Caroline Witton et al $2019 \mathrm{~J}$. Neural Eng. in press https://doi.org/10.1088/1741-2552/ab225e

\section{Manuscript version: Accepted Manuscript}

Accepted Manuscript is "the version of the article accepted for publication including all changes made as a result of the peer review process, and which may also include the addition to the article by IOP Publishing of a header, an article ID, a cover sheet and/or an 'Accepted

Manuscript' watermark, but excluding any other editing, typesetting or other changes made by IOP Publishing and/or its licensors"

This Accepted Manuscript is @ 2018 IOP Publishing Ltd.

During the embargo period (the 12 month period from the publication of the Version of Record of this article), the Accepted Manuscript is fully protected by copyright and cannot be reused or reposted elsewhere.

As the Version of Record of this article is going to be / has been published on a subscription basis, this Accepted Manuscript is available for reuse under a CC BY-NC-ND 3.0 licence after the 12 month embargo period.

After the embargo period, everyone is permitted to use copy and redistribute this article for non-commercial purposes only, provided that they adhere to all the terms of the licence https://creativecommons.org/licences/by-nc-nd/3.0

Although reasonable endeavours have been taken to obtain all necessary permissions from third parties to include their copyrighted content within this article, their full citation and copyright line may not be present in this Accepted Manuscript version. Before using any content from this article, please refer to the Version of Record on IOPscience once published for full citation and copyright details, as permissions will likely be required. All third party content is fully copyright protected, unless specifically stated otherwise in the figure caption in the Version of Record.

View the article online for updates and enhancements. 
Caroline Witton ${ }^{1}$, Sergey V. Sergeyev ${ }^{2}$, Elena G. Turitsyna ${ }^{2}$, Paul L. Furlong ${ }^{1}$, Stefano Seri ${ }^{1}$, Matthew Brookes $^{3}$, Sergei K. Turitsyn ${ }^{2 *}$

6

$7 \quad{ }^{1}$ Aston Brain Centre, Aston University, Birmingham, B4 7ET, UK

8

${ }^{2}$ Aston Institute of Photonic Technologies, Aston University, Birmingham, B4 7ET, UK

9

${ }^{3}$ Sir Peter Mansfield Imaging Centre, University of Nottingham, Nottingham, NG7 2RD, UK

10

Dr. Caroline Witton Aston Brain Centre Aston University, Birmingham B4 7ET

Tel: 01212044087

c.witton@aston.ac.uk

Keywords:

23 Nonlinear dynamics, epilepsy, magnetoencephalography, 
Abstract:

Objective. Brain electromagnetic activity in patients with epilepsy is characterized by abnormal highamplitude transient events (spikes) and abnormal patterns of synchronization of brain rhythms that accompany epileptic seizures. With the aim of improving methods for identifying epileptogenic sources in magnetoencephalographic (MEG) recordings of brain data, we applied methods previously used in the study of oceanic 'rogue waves' and other freak events in complex systems Approach. For data from 3 patients who were awaiting surgical treatment for epilepsy, we used a beamformer source model to produce volumetric maps showing areas with a high proportion of spikes that could be classified as 'rogue waves', and areas with high Hurst Exponent (HE). The HE describes the extent to which a system is exhibiting persistent behavior, may predict the likelihood of freak events. These measures were compared with the more standard measure of kurtosis, which has been shown to be a reliable method for localizing interictal spikes.

Main Results. There was partial concordance between the 3 different volumetric maps indicating that each measure provides different information about the underlying brain data. The HE, when combined with a simple connectivity analysis based on phase slope index, was able to identify the probable epileptogenic zone in all 3 patients, despite very different patterns of abnormal activity.

The differences between distributions of high $\mathrm{HE}$ and high kurtosis values indicates that while spikes are propagated through cortex from the epileptogenic zone, the persistent dynamical conditions under which the spikes are generated may not be propagated in a similar way. Finally, the patterns of persistent activity, indicating a departure from 'healthy criticality' in brain networks may explain the wide range of social and cognitive impairments that are seen in epilepsy patients.

Significance. The HE is a potentially useful addition to the clinician's battery of measures which may be used convergently to guide surgical intervention. 
Epilepsy affects up to $1 \%$ of people worldwide (Fiest et al., 2017) and is characterized by seizures, which are thought to occur because of a disruption to the balance of neuronal excitation and inhibition in the brain (Fisher et al., 2005). These typically take the form of abnormal, strong and synchronised activity beginning within discrete populations of neurons but propagating through wide areas of brain tissue. Many cases of epilepsy respond successfully to pharmacological intervention, but for those which don't, surgical resection of epileptogenic brain tissue can lead to a reduction or even eradication of seizures. The work described here aims to improve methods for identifying epileptogenic sources in magnetoencephalographic (MEG) recordings of brain data, which could ultimately be used to guide surgical intervention.

Recent developments in our understanding of the brain as a nonlinear dynamical system suggest that the healthy brain needs to operate near a state of criticality. In the brain, self-organized criticality depends on the balance of excitation and inhibition between nodes in neural networks, that - in healthy systems - are tuned to allow the occurrence of scale-free 'avalanches' through which information is propagated optimally (Plenz, 2012). This ensures that the system maintains functionally-appropriate dynamic range, fidelity of information processing, and information capacity (Shew and Plenz, 2013). Self-organized criticality is considered a hallmark of behavior in many other naturally-occurring complex systems, from solar flares to epidemics (Bak, Tang and Wiesenfeld, 1987; Jensen, 1998). It is this balance of neural excitation and inhibition that is disrupted in the pathophysiology of epilepsy.

At the macroscopic level, for example on an electroencephalogram (EEG) or in MEG data, epileptiform discharges are typically observed as very frequent, very high-amplitude spike-and-wave complexes, initially within discrete populations of neurons and then propagating through brain networks (de Curtis and Avanzini, 2001). Between seizures (interictally), many epileptic brains will display occasional abnormal discharges, typically but not always spikes, which may originate from the epileptogenic zone, or elsewhere. It has been suggested the spikes arise in the context of a deviation from the normally-observed power-law statistics that are associated with healthy criticality, even between seizures (Acharya et al., 2013; Song and Zhang, 2013; Yan et al., 2016). Large in amplitude and unpredictable, interictal spikes can be likened to the 'rogue' or 'freak' waves which emerge from nonlinear processes in many other dynamical systems - such as on the ocean's surface (Steele, Thorpe and Turekian, 2009) and in nonlinear optics (Onorato et al., 2013).

The Hurst exponent (HE) has emerged as a useful metric for predicting the likelihood of freak events (Feder, 1988; Grech and Mazur, 2004; Gao et al., 2007; Resta, 2012; Eftaxias et al., 2013). The rescaled range method for estimating the HE was originally developed in the 1950s for the study of 
long-range dependence in water storage, in the River Nile (Anis and Lloyd, 1976; Nolte et al., 2008). The HE reflects the change in autocorrelation in a time series over successive time-lags; a slow, power-law, decay in autocorrelation yields a HE value between 0.5 and 1 and indicates the presence of persistent statistics (e.g. a trend for growth for one time interval is more likely to be followed by a trend for growth in the following time interval). Higher $\mathrm{HE}$ is also associated with a higher likelihood of freak events. A HE value between 0 and 0.5 indicates the presence of antipersistence, characterized by wild fluctuations in the data. A time series which is Gaussian noise, i.e. a random walk, will have a HE of 0.5.

The HE has been applied to epilepsy previously, in the context of automatic detection of spike and seizure activity in EEG timeseries, using different methods, e.g. rescaled range analysis (Blythe et al., 2014), detrended fluctuation analysis and wavelet-transform based techniques (Madan et al., 2018). It has also been applied recently in other brain disorders associated with disordered cognition, such as PTSD (Rahmani et al., 2018) and concussion (Munia et al., 2017). Here we extend the application of the HE in epilepsy work, by demonstrating how it can be used in source modelling of MEG data, creating a potential tool for clinicians planning surgery to treat pharmaco-resistent epilepsy.

To aid in presurgical evaluation, a neuroimaging approach needs to be able to identify epileptiform activity, and to produce some kind of volumetric image that can be coregistered with anatomical images such as MR images and used in concert with clinical neuronavigation systems. The traditional approach for localizing the source of epileptiform activity in MEG data has been manual identification and fitting of equivalent-current dipolés to individual or averaged spikes (e.g. Ochi and Otsubo, 2008). However beamformer-based methods which can effectively scan the whole brain volume for epileptic discharges are of increasing interest. The underlying beamformer source model (van Veen et al., 1997; Sekihara et al., 2002; Brookes et al., 2008) has the benefits of significantly improved SNR and of requiring no a priori assumptions about the number of sources, compared to traditional dipole-fitting methods (Adjamian et al. 2009; Hoogenboom et al. 2006). The standard beamformer-based spike localisation method measures kurtosis: if a voxel's data contains spikes, the distribution of its pooled timeseries will often be kurtotic because of the occurrence of rare-highamplitude discharges (Kirsch et al., 2006; Hall et al., 2018). This is akin to the 'heavy tailed' distributions that are characteristic of systems exhibiting rogue waves (Onorato et al., 2013), where freak events are more common than predicted by a normal distribution. But although the kurtosis measure identifies a heavy tail, it does not determine whether the system is exhibiting persistent behaviour. Conversely, the HE can reflect the situation where the likelihood of freak events, such as spikes, is coupled with persistence and power-law behaviour, indicating a deviation from healthy criticality. 
119 The aim of the present study was to determine whether the HE is able to identify beamformer voxels 120 whose timeseries contain abnormal, epileptiform, brain activity; and how this might complement measures of kurtosis or simple rogue waves, both interictally and during the time surrounding a seizure. Connectivity analysis on putative epileptogenic networks derived from the HE maps are also explored in the context of clinical outcomes.

124

\section{Methods}

\section{$126 \quad$ Case Studies}

127 The case studies are based on data from three patients with pharmaco-resistant epilepsy, who had 128 been referred to the Aston Brain Centre for assessment using MEG as part of their clinical evaluation 129 prior to resection surgery for treatment of seizures. The patients' details are/shown in Table 1.

130 The data described below are from the patients' routine pre-surgical MEG recordings, which were 131 analysed clinically using standard kurtosis beamformer methods and interpreted alongside evidence 132 from corticography, EEG, and structural MR imaging. All patients underwent surgery, and the 133 outcomes described in Table 1 are from long term ( $>5$ year) clinical follow-ups done post-surgically. 134 All work was conducted in accordance with the Declaration of Helsinki and with the approval of the local Ethics Committee.

MEG Recordings

MEG data were recorded using a 275-channel whole-head CTF MEG system (CTF Systems, Port Coquitlam, Canada) with synthetic third-order gradiometers (Vrba et al., 1999) sampled at $1200 \mathrm{~Hz}$ with an anti-aliasing filter of $600 \mathrm{~Hz}$, and de-trended to correct for baseline drift. Each MEG dataset was spatially co-registered with the individual's T1-weighted structural MRI using a modification of the surface-matching method described by Adjamian et al. (Adjamian et al., 2004), and a multisphere head model (Huang, Mosher and Leahy, 1999) was derived from each participant's brain surface. Data were band-pass filtered between 1 and $150 \mathrm{~Hz}$ and projected into source space using a scalar beamformer (Robinson and Vrba, 1999). Beamformers use the covariance matrix of the data to create a set of weights for each point in a grid of voxels across the volume of the brain, which can be used to produce volumetric images and to reconstruct spatially-filtered source timeseries. 
150

151

152

153

154

155

156

157

158

159

160

161

162

163

164

165

166

167

168

169

170

171

172

173

174 The rescaled range is found as $R_{N} / S_{N}$. As a next step, time series of $N$ points were divided in two $N / 2$ -

175 point time series and the rescaled range $R_{N / 2} / S_{N / 2}$ was calculated for both time series and further

176 averaged. The process was repeated for partial series which comprised $n=N / 4, N / 8 \ldots$ points. 
177

$$
E\left(R_{n} / S_{n}\right)=C n^{H E}
$$

182 Here $\mathrm{E}(\mathrm{x})$ is the expected value and $\mathrm{C}$ is a constant.

\section{$\underline{\text { Rogue waves analysis }}$}

To calculate the percentage of rogue waves, we used the same pragmatic approach used in

classifying oceanic rogue waves, i.e. whenever the wave height $H$ exceeded twice the significant wave height $H_{\mathrm{s}}$. Traditionally, the significant wave height was defined as the average of the one-third

\section{Connectivity analysis}

Connectivity between timeseries for voxels with peak HE values was assessed using the Phase Slope Index (PSI) (Nolte et al., 2008), using the Matlab function adapted by Cohen, 2014 (Cohen, 2014). PSI determines whether the slope of the phase lag across frequencies between timeseries is consistently positive or negative, with positive values between areas A and B indicating that the activity in area $\mathrm{A}$ is driving the activity in area $\mathrm{B}$, either directly or indirectly. Its non-parametric approach makes PSI particularly appropriate for data, such as these, that do not meet the requirements of the typical vector autoregressive (VAR) model underpinning other methods for estimating directional connectivity, such as Granger causality. (VAR models typically expect the autocorrelation of the data to decay exponentially (Barnett and Seth, 2014), whereas our analysis 204 based on the Hurst Exponent illustrates that this decay actually approaches a power-law function). 
205 PSI values are expressed as standard deviations against a randomised population distribution for the

206 null hypothesis, derived from 1000 permutations. For our data, PSI was computed over a broad

207 frequency band of 1-150 Hz (the same band as used for the HE analysis); and for a classically-defined

208 EEG 'theta-to-beta' band $(4-30 \mathrm{~Hz})$. For patients 1 and 2, similar effects were found in both

209 frequency bands so the broad band results are reported. For patient 3, pre-seizure effects were

210 predominantly constrained to the lower $4-30 \mathrm{~Hz}$ band, so only these values are reported. In the

211 figures, PSI values exceeding 1.96 standard deviations are shown, to indicate directional influences

212 exceeding the $95 \%$ confidence interval on the assumption of a normal distribution for a 1-tailed test.

213 Tables show all PSI values for each patient.

214

215 


\section{Results}

217 Volumetric images mapping the HE and percent rogue waves for each patient were compared with 218 the images showing the cortical distribution of kurtosis values, an accepted measure of interictal 219 spiking activity (Kirsch et al., 2006; Hall et al., 2018). Figure 1 shows an example slice for each 220 patient, from images that were thresholded to include only the highest $2.5 \%$ of values. In all cases,

221 the images show a wide, bilateral, network of areas with putative epileptiform activity. Clearly,

222 although spike activity may propagate through such wide networks, further analysis and clinical

223 interpretation are required to determine which part of the network corresponds to the

224 epileptogenic zone. This may be done through convergent analyses, and examination of source

225 timeseries including connectivity estimation.

226 The images in Figure 1 overlap, but not completely - indicating that the measures are not

227 interchangeable. Table 2 shows the percentage overlap between the 3 different image types for the 228 patients, and, for reference, the concordance between the independent images across the three 229 patients. In the case of the rogue waves and kurtosis images for Patient 1, the concordance 230 between images is high (77\%). But in all other cases, the overlap is comparable to the comparison across patients, ranging between $16 \%$ and $48 \%$ (Table 2). The measures are clearly reflecting different aspects of the data, and it is important to determine whether the potentially epileptogenic sources are reflected only in the overlapping zones, or whether the HE measure provides new, clinically relevant information, for each patient.

235 Figure 2 shows further data for Patient 1. The maximum cortical HE value was 0.82, with HE values 236 showing a clear topographical pattern with several local peaks. One peak occurred in the left 237 temporal cortex, where the three images overlapped (Fig. 2a). Figure 2b shows the timeseries from 238 this location, with the 21 spikes meeting criteria for rogue waves identified on the plot. Fig. 2c shows 239 the probability density function for the squared timeseries upon which the HE and rogue waves 240 measures were computed, with the extreme values in the tail that form the basis of the concordant 241 kurtosis and rogue waves images at this location. Figures $2 \mathrm{~d}$ and $2 \mathrm{e}$ show the spikes occurring just 242 after 30 seconds in more detail, in both the time (2d) and frequency (2e) domains (See also SI Movie 2431 for an animated spectrogram illustrating the spectral power changes associated with the rogue 244 waves).

245 Importantly, though, spikes are not seen at all locations with high HE values. Figure $2 \mathrm{f}$ shows the 246 data around 30 seconds again, with timeseries from the top four peaks in the HE image and one 247 from a control voxel with lower HE. While a clear spike is also seen in the left frontal source, 248 simultaneously with the temporal source but with opposite polarity; the right occipital and parietal 
249

250

251

252

253

254

255

256

257

258

259

260

261

262

263

264

265

266

267

268

269

270

271

272

273

274

275

276

277

278

279

280

281

sources identified by the HE map do not show a spike at this time. There, the high $\mathrm{H}$ values are not always accompanied by rogue waves, and this dissociation can explain the relatively low concordance between the images based on HE and those based on extreme values, i.e. kurtosis and rogue waves.

A measure of phase slope index (Nolte et al., 2008; Cohen, 2014) across a broad frequency range of 1-150 $\mathrm{Hz}$ was used to estimate the presence of significant directed communication between the nodes in this network (Fig $2 \mathrm{~g}$ ). The left temporal source is a clear driver of activity in the left frontal, right parietal and right occipital areas that also show strong persistent statistics (and is not a significant driver of activity in the control region that did not show a peak in the $\mathrm{H}$ map). PSI values are given in Table 3.

This network analysis of regions identified by our $\mathrm{H}$ measure was confirmed during the subsequent pre-operative evaluation for this patient, which identified a location in the left temporal lobe as the epileptogenic zone. Its surgical removal resulted in complete freedom from seizures. Thus, for this patient, the $\mathrm{HE}$, rogue waves, and kurtosis images were all able to indicate the epileptogenic source, which lay in the region with high concordance between measures.

Data for the second patient are shown in Figure 3. HE values across the cortex were higher overall than in Patient 1, with much of the cortex showing HE values above 0.75 ; the thresholded image contains values between 0.8 and 0.85 . The overlap between the HE image and the kurtosis and rogue waves images is low (Table 2), with a left ventrolateral frontal area being the main area of concordance between HE and kurtosis (e.g. as shown in Fig. 3a). Clusters of high amplitude spikes can be seen at this location in Fig. 3b, and the probability density function in $3 c$ shows a clear 'heavy tail' corresponding to the presence of high-amplitude events here. (See also SI Movie 2 for an animated spectrogram showing bursts of spectral power associated with the rogue waves). This patient had a neuroanatomical abnormality in the right parietal lobe (Fig. 3d), the area surrounding which was a clinically-likely epileptogenic source. However the only measure to show activation that survived thresholding in this region was the measure of rogue waves (Fig. $3 \mathrm{~d}$ ).

The right parietal area adjacent to the lesion, along with the left ventrolateral frontal area of overlap and the two other highest peaks in the HE image were selected for further analysis, alongside a control region with low HE. Figure $3 e$ shows the timeseries for each location around the largest spike complex occurring just after 50 seconds. Spikes originating in the right parietal and left ventrolateral frontal sources are of substantially higher amplitude than those at the left frontal location, and very little abnormal activity is observable by eye at the occipital location despite its relatively high HE values above 0.8 ; the control region also contains spikes. There is also evidence of spike activity 
282

propagating through into the control timeseries where the HE was relatively low, although still exceeding 0.75 .

Timeseries from all five loci were entered into a connectivity analysis. Phase-slope analysis identified two regions which were significant, independent drivers of activity in the other areas in this patient: the right parietal region and the left ventrolateral frontal region (Fig 3f; Table 4). Although both were shown to drive activity in the frontal and occipital areas identified from the HE image, and the control area, there was no significant directed connectivity between these two areas.

This patient subsequently underwent surgery to resect tissue from the right parietal area of anatomical abnormality. However this had limited success and resulted only in a reduction in seizure frequency for the patient. Thus the left ventrolateral frontal region reflected by the overlapping $\mathrm{HE}$ and kurtosis measures is the likely driver of this patient's remaining seizures.

Figure 4 shows data from a third patient who had a seizure during the MEG recording. In Figure 4a, the maps showing $\mathrm{HE}$, kurtosis, and percent rogue waves are compared for an example slice, for the 7 seconds of data before the onset of the seizure. The HE image gives a markedly different topography of abnormal activity compared to the kurtosis and rogue waves images (15.6\% and $29.6 \%$ overlap respectively). The kurtosis and rogue waves images are more similar (48.1\% overlap). Figure $4 \mathrm{~b}$ shows the timeseries of the whole recording, reconstructed from the right frontal source visible in the HE map in 4a (the location that was later determined to be the epileptogenic source). Note the absence of spikes in the pre-seizure data at this location, despite the extremely high $\mathrm{HE}$ values. Figure $4 c$ shows a spectrogram of data for this pre-ictal time period, illustrating that abnormal activity is restricted to somewhat lower frequencies than typically characterized by spikes (for example, compare Fig. 4c with Fig. 2e). During the seizure, very large strongly synchronous activity is seen (Fig. 4b) and the maximum HE value in the image shown in Figure 4d from the data during the seizure has dropped from 1 to 0.88 . Following the seizure, the maximum HE value remains lower (0.86; Figure 4e). During and after the seizure the topographic patterns of HE values change substantially compared to the map from before the seizure. (See also SI Movie 3 which shows spectral power before, during, and after the seizure).

There were three local peaks in the thresholded pre-seizure HE image, for which a slice is shown in Figure 4a; in the right frontal; left temporal, and right superior parietal cortex. Timeseries for the data before the seizure for each of these loci were entered into a connectivity analysis. The broad band phase-slope analysis (as used for Patients 1 and 2) did not reveal significant connectivity within this patient's pre-seizure data; however an analysis restricted to the classical theta, alpha and beta frequency bands did reveal significant driving influences from the source in the right frontal lobe to 
315 the left temporal and right superior parietal other sites (Figure $4 e$ and Table 5) The right frontal 316 source was confirmed post-surgically to be the epileptogenic zone in this patient.

317 


\section{Discussion}

319 The aim of these three case studies was to determine whether the Hurst exponent is able to identify

320 beamformer voxels whose timeseries contain abnormal, epileptiform brain activity; and to

321 determine how this analysis might complement the more traditional measure of kurtosis, both

322 interictally and during the time surrounding a seizure. Taken together, the results suggest that the

323 HE can provide significant additional information to the clinical analysis of pre-surgical MEG data.

324 However it is also clear that the HE measure is providing different, complementary, information to

325 the more standard analytical approaches such as kurtosis.

326 Peaks in HE images predicted surgically-confirmed epileptogenic sources in Patients 1 and 3, when

327 entered into a connectivity analysis. In Patient 2, the HE image and connectivity analysis identified a

328 likely additional epileptogenic source that may explain unexpected additional seizures post-surgicaly.

329 In both Patients 1 and 2, this putative epileptogenic zone fell in an area where high HE values co-

330 occurred with spikes, and was thus also identified through the kurtosis image. This observation is

331 consistent with our original hypothesis that the HE measure is a good predictor of the likelihood of

332 'freak events', i.e. spikes (Feder, 1988; Grech and Mazur, 2004; Gao et al., 2007; Resta, 2012;

333 Eftaxias et al., 2013).

334 Importantly, the HE measure is providing a different kind of information to standard measures such

335 as kurtosis. For example in each patient there were other regions of cortex which showed high

336 kurtosis values but where HE values fell below threshold, as illustrated by the low percentage of

337 overlap between images - but these regions did not coincide with the epileptogenic zone. Thus

338 those areas, despite the presence of spikes, were not among those with the greatest tendency

339 towards persistent or super-critical activity. Similarly there were areas, i.e., in the non-overlapping

340 parts of the images, where high HE values did not coincide with high values of kurtosis. In our

341 patients these areas did not represent the epileptogenic zone, Further, in Patient 3, the pre-seizure

342 activity in the epileptogenic zone itself did not contain large spikes (or elsewhere; compare the max

343 kurtosis value of 7.5 in this patient with 23.0 in Patient 2) but had a high HE value. It is possible

344 that, while spikes are propagated through cortex from the epileptogenic zone, the persistent

345 dynamical conditions under which the spikes are generated are not propagated in a similar way,

346 resulting in the markedly different topographical maps that we found.

347 These findings, that areas of high HE form part of a wider network characterized by persistent

348 statistics, echo some reported previously for recordings taken from the cortical surface of patients

349 with epilepsy (ECoG data; (Yan et al., 2016)), where increased HE values were seen just before the 350 onset of a seizure, with persistent dynamics observed beyond the epileptogenic area. Our data, 
351 which includes coverage of the whole cortex rather than a limited number of electrode sites and

352

353

354

355

356

357

358

359

360

361

362

363

364

365

366

367

368

369

370

371

372

373

374

375

376

377

378

379

380

381

382 thus allowing whole-brain network analysis, shows that the pattern of persistent dynamics both interictally and just preceding a seizure forms part of a complex network through which the anomalous spikes are propagated.

The fact that the HE map was able to identify the epileptogenic zone in Patient 3, despite the absence of large spikes in this area, indicates that the HE might be particularly useful for the approximately $30 \%$ of patients who are referred for pre-surgical MEG evaluation but do not show interictal spikes per se. The typical methods used for localizing epileptiform activity, i.e. dipole modelling and kurtosis, are optimized for brief signals with high amplitude. Yet the kind of persistent behavior that is identified in timeseries by the Hurst exponent may be present in some patients' interictal data even in the absence of extremely large transients, meaning that the $\mathrm{HE}$ would still be able to identify epileptogenic sources. Methods which can help localize slower and lower-amplitude abnormalities are a potentially powerful addition to the clinician's toolbox.

The timeseries plots in Figures $2 \mathrm{~b}$ and $3 \mathrm{~b}$ confirm that epileptiform spikes meet the standard oceanographic criteria for 'rogue waves' (Steele, Thorpe and Turekian, 2009). However the measure of percent rogue waves also appears to have a different sensitivity profile than the traditional metric of kurtosis, as the overlap between volumetric images is only partial. This is more surprising than the dissociation between HE and kurtosis, as both aim to identify spikes based on distributional statistics. The difference presumably occurs because of the rogue waves measure's different arithmetic relationship to the standard deviation of the sample points in the timeseries, as the number of spikes increases. Although the kurtosis measure is a reliable indicator of spiking activity (Kirsch et al., 2006; Hall et al., 2018), it can fail in situations where very large numbers of spikes result in a distribution that is not kurtotic because the high values are no longer 'extreme'. Concordance between these measures is lowest for Patient 2, who showed particularly strong and regular spiking activity. In fact, here the percent rogue waves map was the only one of our thresholded images to identify the parietal zone that was clinically selected for this patient's original, but only partially successful surgery. This new approach therefore also has potential as an additional tool for localization of spikes in cases where kurtosis fails and is worthy of further study in a clinical trial which does not have the limited sample size of the current set of case-studies.

It is also noteworthy that for Patient 2, the parietal epileptogenic zone that was revealed by the rogue waves analysis had low HE (whereas their presumed secondary epileptogenic zone had high $\mathrm{HE}$ ), illustrating that not all epileptogenic zones might be identified on the basis of the Hurst

383 exponent. The general observation that the different measures all have different sensitivity profiles 
in the face of varying data properties underlines the potential value of approaches which use information from more than one analysis method to triangulate upon the epileptogenic source. This idea is also reflected in work that has used the Hurst exponent alongside several other complementary measures in automatic classifiers for the detection of epileptic activity (e.g. Acharya et al., 2013)

Our connectivity analysis based on phase-slope index, a non-parametric index of directed connectivity (Nolte et al., 2008; Cohen, 2014), offers a final potential tool for clinical application. The observation that epileptiform timeseries show strongly persistent activity, as indexed by the $\mathrm{HE}$, means that the typical vector autoregressive models used to underpin Grainger causality, for example, are not suitable for epilepsy data. The phase-slope index is a simple alternative that works well in timeseries characterized by broad-band bursts of power associated with epileptiform data.

The results described here present the first whole-brain analysis of epilepsy data based on the Hurst Exponent and demonstrate the potential for such nonlinear dynamical approaches as an additional tool for clinicians undertaking pre-surgical investigations, as well as the possibilities for the rogue waves and phase-slope analyses which are based on the same source model. However this is not a clinical trial and therefore the next appropriate step is for a clinical trial to be conducted, with a larger population of patients and a systematic evaluation of the efficacy of these measures in comparison with existing clinical protocols.

Overall, our findings demonstrate the clear presence of persistent statistics, indicating a departure from healthy self-organized criticality, in epileptic brains both well between seizures and just before the onset of a seizure. The complex topographic distributions of high Hurst exponents in these patients and associated connectivity patterns indicate that this super-critical state can be propagated, as can the spikes themselves, through a range of cortical areas form the origin of the abnormal, persistent activity. If a state of near-criticality is required for efficient information processing then the high Hurst exponent value seen in our patients may explain the wide range of cognitive impairments and neurodevelopmental disorder seen in patients with epilepsy, especially children.

Our findings, apart from the interest for bio-medicine, can provide a base for developing new Hurst $\mathrm{R} / \mathrm{S}$ analysis-based predictors of the emergence and localization of the rogue/freak waves in different complex networks (Internet, power grids, financial and logistic systems, and other applications.) 


\section{References}

419 Acharya, U. R. et al. (2013) 'Automated EEG analysis of epilepsy: A review', Knowledge-Based

420 Systems, 45, pp. 147-165. doi: 10.1016/j.knosys.2013.02.014.

Adjamian, P. et al. (2004) 'Co-registration of magnetoencephalography with magnetic resonance imaging using bite-bar-based fiducials and surface-matching.', Clinical neurophysiology : official journal of the International Federation of Clinical Neurophysiology, 115(3), pp. 691-8. doi: 10.1016/j.clinph.2003.10.023.

Anis, A. A. and Lloyd, E. H. (1976) 'The expected value of the adjusted rescaled Hurst range of independent normal summands', Biometrica, 63, pp. 283-298.

Bak, P., Tang, C. and Wiesenfeld, K. (1987) 'Self-organized criticality: An explanation of the $1 / f$ noise', Physical Review Letters. American Physical Society, 59(4), pp.381-384. doi: 10.1103/PhysRevLett.59.381.

Barnett, L. and Seth, A. K. (2014) 'The MVGC multivariate Granger causality toolbox: A new approach to Granger-causal inference', Journal of Neuroscience Methods, 223, pp.50-68. doi: 10.1016/j.jneumeth.2013.10.018.

Blythe, D. A. J. et al. (2014) 'The effect of linear mixing in the EEG on Hurst exponent estimation', Neurolmage, 99, pp. 377-387. doi: 10.1016/j.neuroimage.2014.05.041.

Brookes, M. J. et al. (2008) 'Optimising experimental design for MEG beamformer imaging.', 436 Neurolmage, 39(4), pp. 1788-802. doi: 10.1016/j.neuroimage.2007.09.050.

437 Cohen, M. X. (2014) 'Analyzing neural time series data: Theory and practice'. neurobiology, 63(5), pp. 541-67. Available at: http://www.ncbi.nlm.nih.gov/pubmed/11164621 (Accessed: 21 June 2017).

441 Eftaxias, K. et al. (2013) 'Dynamical analogy between epileptic seizures and seismogenic 442 electromagnetic emissions by means of nonextensive statistical mechanics', Physica A: Statistical 443 Mechanics and its Applications, 392(3), pp. 497-509. doi: 10.1016/j.physa.2012.09.015.

444 Feder, J. (1988) Fractals. New York and London: Plenum Press.

Fiest, K. M. et al. (2017) 'Prevalence and incidence of epilepsy: A systematic review and metaanalysis of international studies.', Neurology, 88(3), pp. 296-303.

447 Fisher, R. S. et al. (2005) 'Epileptic Seizures and Epilepsy: Definitions Proposed by the International 448 League Against Epilepsy (ILAE) and the International Bureau for Epilepsy (IBE)', Epilepsia. Blackwell 449 Science Inc, 46(4), pp. 470-472. doi: 10.1111/j.0013-9580.2005.66104.x.

450 Gao, J. et al. (2007) Multiscale Analysis of Complex Time Series: Integration of Chaos and Random 451 Fractal Theory, and Beyond. WILEY-BLACKWELL.

452 Grech, D. and Mazur, Z. (2004) 'Can one make any crash prediction in finance using the local Hurst 453 exponent idea?', Physica A: Statistical Mechanics and its Applications, 336(1-2), pp. 133-145. doi: 454 10.1016/j.physa.2004.01.018.

455 Hall, M. B. H. et al. (2018) 'An evaluation of kurtosis beamforming in magnetoencephalography to 
456 localize the epileptogenic zone in drug resistant epilepsy patients', Clinical Neurophysiology, 129(6).

457 doi: 10.1016/j.clinph.2017.12.040.

458 Huang, M. X., Mosher, J. C. and Leahy, R. M. (1999) 'A sensor-weighted overlapping-sphere head 459 model and exhaustive head model comparison for MEG.', Physics in medicine and biology, 44(2), pp. 460 423-40. Available at: http://www.ncbi.nlm.nih.gov/pubmed/10070792 (Accessed: 24 January 2014).

461 Jensen, H. J. (1998) Self-organized criticality : emergent complex behavior in physical and biological 462 systems. Cambridge University Press.

463 Kirsch, H. E. et al. (2006) 'Automated localization of magnetoencephalographic interictal spikes by 464 adaptive spatial filtering', Clinical Neurophysiology, 117(10), pp. 2264-2271. doi:

465 10.1016/j.clinph.2006.06.708.

466 Madan, S. et al. (2018) 'A case study on Discrete Wavelet Transform based Hurst exponent for 467 epilepsy detection', Journal of Medical Engineering \& Technology, 42(1), pp. 9-17. doi: 468 10.1080/03091902.2017.1394390.

469 Munia, T. T. K. et al. (2017) 'A Novel EEG Based Spectral Analysis of Persistent Brain Function 470 Alteration in Athletes with Concussion History', Scientific Reports. Nature Publishing Group, 7(1), p. 471 17221. doi: 10.1038/s41598-017-17414-x.

472 Nolte, G. et al. (2008) 'Robustly Estimating the Flow Direction of Information in Complex Physical 473 Systems', Physical Review Letters. American Physical Society, 100(23), p. 234101. doi: 474 10.1103/PhysRevLett.100.234101.

475

476

477

478

479

480

481

482

483

484

485

486

487

488

489

490

491

492

493

494

495

496

497

498

Ochi, A. and Otsubo, H. (2008) 'Magnetoencephalography-guided epilepsy surgery for children with intractable focal epilepsy: SickKids experience.', International journal of psychophysiology : official journal of the International Organization of Psychophysiology, 68(2), pp. 104-10. doi: 10.1016/j.ijpsycho.2007.12.008.

Onorato, M. et al. (2013) 'Rogue waves and their generating mechanisms in different physical contexts', Physics Reports, 528(2), pp. 47-89. doi: 10.1016/j.physrep.2013.03.001.

Peters, E. E. (1994) Fractal Market Analysis: Applying Chaos Theory to Investment and Economics. 1st Editio. New York: Wiley.

Plenz, D. (2012) 'Neuronal avalanches and coherence potentials', The European Physical JournalSpecial Topics, 205, pp. 259-301. Available at:

http://www.springerlink.com/index/5076JJ82172077R8.pdf (Accessed: 2 May 2017).

Rahmani, B. et al. (2018) 'Dynamical Hurst analysis identifies EEG channel differences between PTSD and healthy controls', PLOS ONE. Edited by L. Jäncke, 13(7), p. e0199144. doi: 10.1371/journal.pone.0199144.

Resta, M. (2012) 'Hurst Exponent and its Applications in Time-series Analysis', Recent Patents on Computer Science, 5(211-219).

Robinson, S. and Vrba, J. (1999) 'Functional neuroimaging by synthetic aperture magnetometry (SAM).' , in Yoshimoto, T. et al. (eds) Recent Advances in Biomagnetism. Tohoku, Japan: Tohoku University Press.

Sekihara, K. et al. (2002) 'Performance of an MEG adaptive-beamformer technique in the presence of correlated neural activities: effects on signal intensity and time-course estimates.' , IEEE Transactions on Biomedical Engineering, 49(12 Pt2), pp. 1534-46. Available at: http://www.ncbi.nlm.nih.gov/pubmed/12549735 (Accessed: 7 February 2014). Shew, W. L. and Plenz, D. (2013) 'The Functional Benefits of Criticality in the Cortex', The 
499 Neuroscientist, 19(1), pp. 88-100. doi: 10.1177/1073858412445487.

500 Song, Y. and Zhang, J. (2013) 'Automatic recognition of epileptic EEG patterns via Extreme Learning

501 Machine and multiresolution feature extraction', Expert Systems with Applications, 40(14), pp. 5477502 5489. doi: 10.1016/j.eswa.2013.04.025.

503 Steele, J. H., Thorpe, S. A. and Turekian, K. K. (eds) (2009) Elements of Physical Oceanography: A 504 derivative of the Encyclopedia of Ocean sciences. 1st Editio. Academic Press.

505 Veen, B. D. Van et al. (1997) 'Localization of Brain Electrical Activity via Linearly Constrained 506 Minimum Variance Spatial Filtering', IEEE Transactions on Biomedical Engineering, 44(9), pp. 867507880.

508 Vrba, J. et al. (1999) '151-Channel whole-cortex MEG system for seated or supine positions', in 509 Yoshimoto, T. et al. (eds) Recent Advances in Biomagnetism. Sendai: Tohoku University Press, pp. 510 93-96.

511 Weron, R. (2002) 'Estimating long-range dependence: finite sample properties and confidence 512 intervals', Physica A: Statistical Mechanics and its Applications, 312(1-2), pp. 285-299. doi: 513 10.1016/S0378-4371(02)00961-5.

514 Yan, J. et al. (2016) 'Analysis of electrocorticogram in epilepsy patients in terms of criticality', 515 Nonlinear Dynamics. Springer Netherlands, 83(4), pp. 1909-1917. doi: 10.1007/s11071-015-2455-9.

516

517 Acknowledgments.

518 The Aston Brain Centre is supported by the Dr Hadwen Trust for Humane Research. S.V.S.

519 acknowledges support from the Leverhulme Trust (Grant ref: RPG-2014-304). S.K.T, S.V.S and E.G.T. 520 acknowledge support from the H2020-MSCA-RISE-20165 project CARDIALLY. 
Figure 1
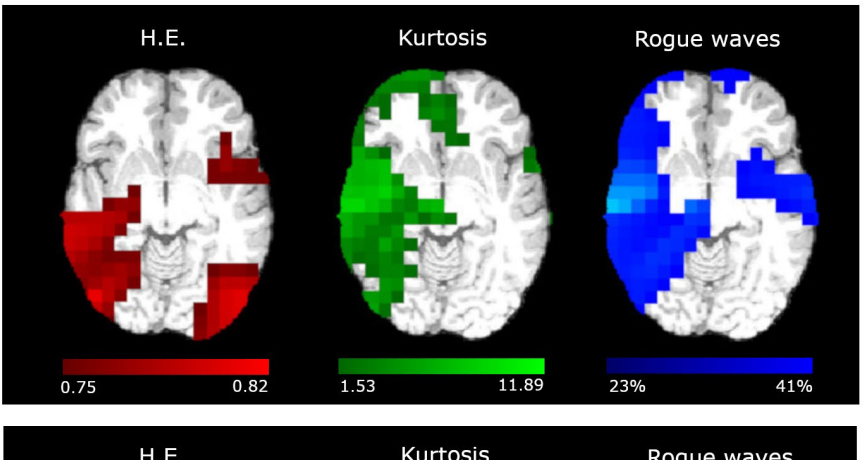

Rogue waves
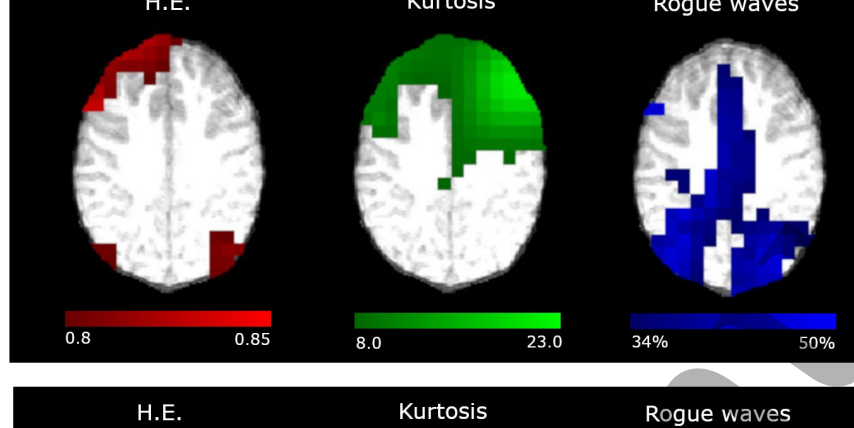

Rogue waves
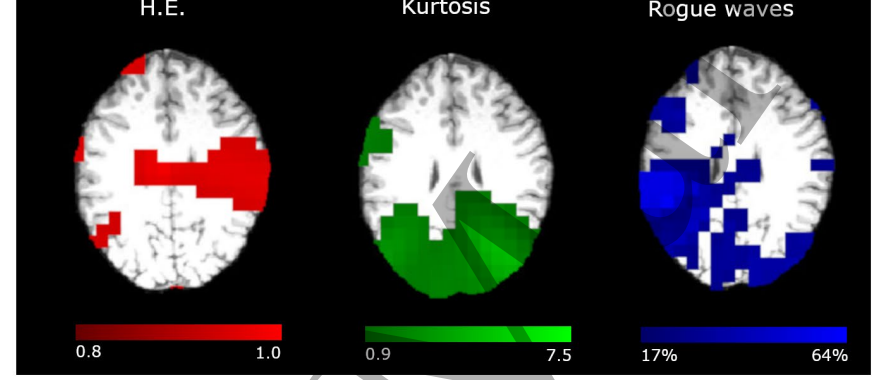

524

525

Figure 1

526 An example slice from Patient 1 (top) and Patient 2 (centre) and Patient 3 (bottom), showing the 527 activity maps based on the HE, kurtosis, and percent rogue waves. The images are thresholded so 528 that only activity in the highest ranked $2.5 \%$ of voxels is shown. Images for Patient 3 are based on 529 the pre-seizure activity only; see Figure 4 for details.

530

531 

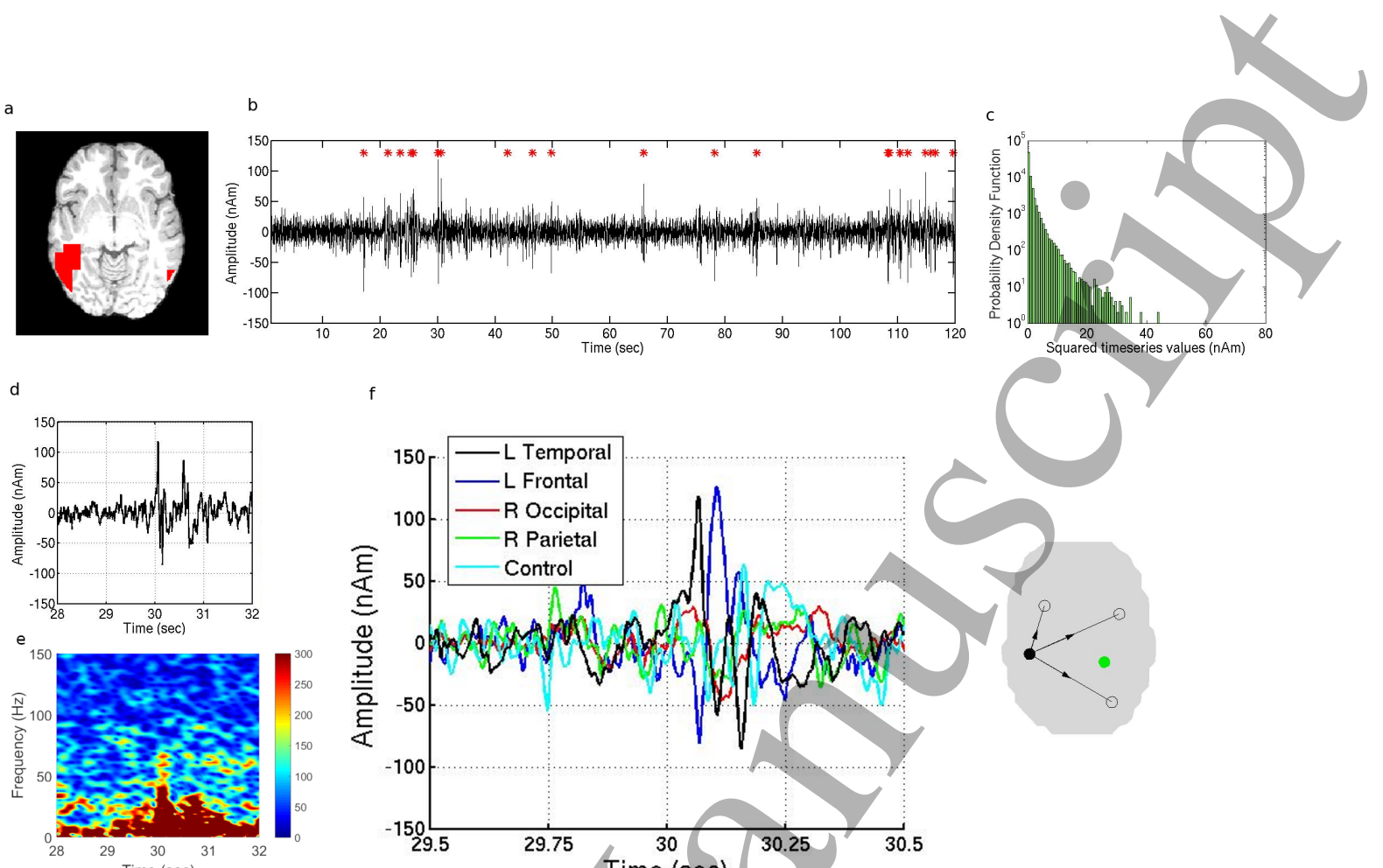

f

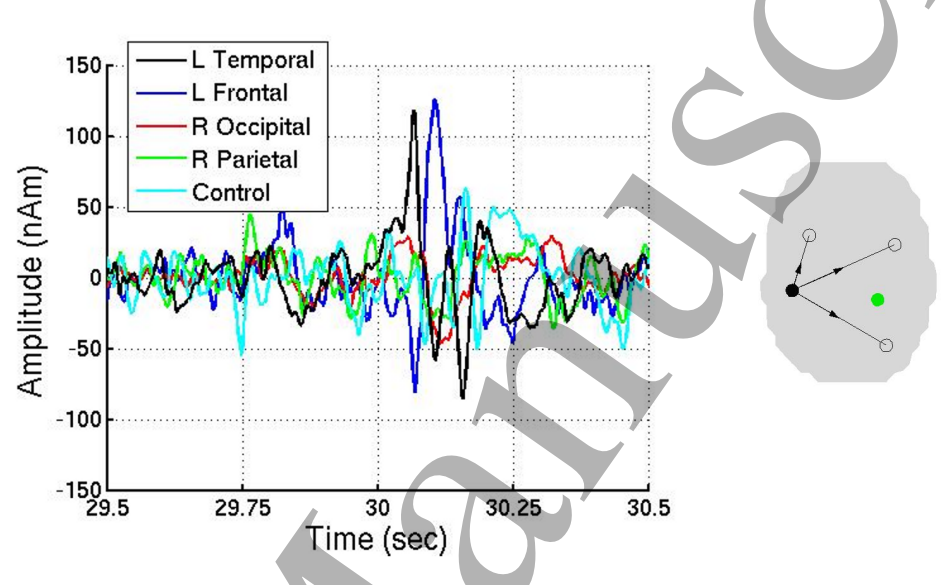

534

Figure 2

Data for Patient 1. Figure 2a shows the area of overlap between the thresholded HE and kurtosis maps for this patient, in one example slice. Fig. $2 \mathrm{~b}$ shows the full data timeseries reconstructed at a voxel in the left temporal area of overlap seen in 2a. Spikes which meet criteria for rogue waves are identified with a red asterisk. Fig. $2 c$ shows the probability density function of values in this timeseries, squared as for the calculation of HE values. Fig. $2 \mathrm{~d}$ shows a zoomed image of the timeseries for a single spike at this same location, occurring just after 30 seconds and Fig. 2e a spectrogram of the same data. The same spike is shown again in Fig. $2 \mathrm{f}$, with the equivalent timeseries for four other locations in different colours: three other peaks in the HE image, and a control location with low $\mathrm{HE}$. The pattern of directed connectivity between the five areas, based on phase slope index, is illustrated on an outline of the downsampled brain in Figure $2 \mathrm{~g}$. The five locations are indicated, with the control area shown in green. Lines with arrows represent the direction of connectivity, and the filled circle indicates the left temporal site which corresponds to the driving source. 

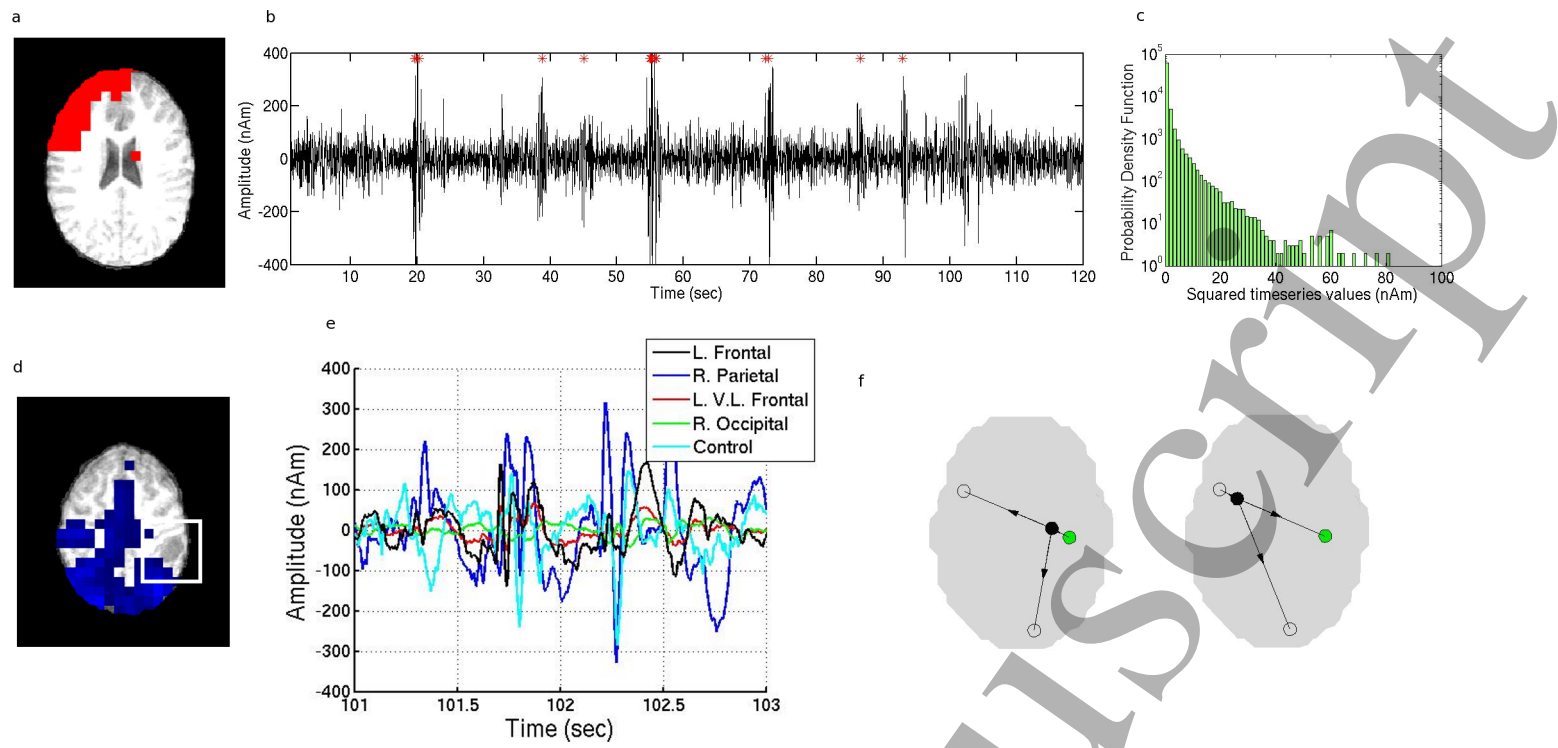

\section{Figure 3}

Data for Patient 2. Figure 3a shows the area of overlap between the thresholded HE and kurtosis maps for this patient, in one example slice. Figure $3 b$ shows the full data timeseries reconstructed at a voxel in the left ventrolateral frontal area of overlap seen in 3a. Spikes which meet criteria for rogue waves are identified with a red asterisk. Figure $3 c$ shows the probability density function of values in this timeseries, squared as for the calculation of HE values. Figure $3 \mathrm{~d}$ shows a slice from the volumetric image showing percent rogue waves. The white box highlights the neuroanatomical lesion which was chosen for resection, alongside an area of high percent rogue waves. Figure $3 e$ shows a zoomed image of the timeseries for all voxels of interest at 101-103 seconds, a time when spiking activity was observed in the left ventrolateral frontal voxel. Figure $3 \mathrm{f}$ shows two connectivity maps, illustrating the patterns and directions of connectivity between from the two sources that were shown by the PLI analysis to be driving activity at the other voxels: the right parietal and left ventrolateral frontal sources. In each case the driving sources are represented by black filled circles, and control sources are shown in green. 
572
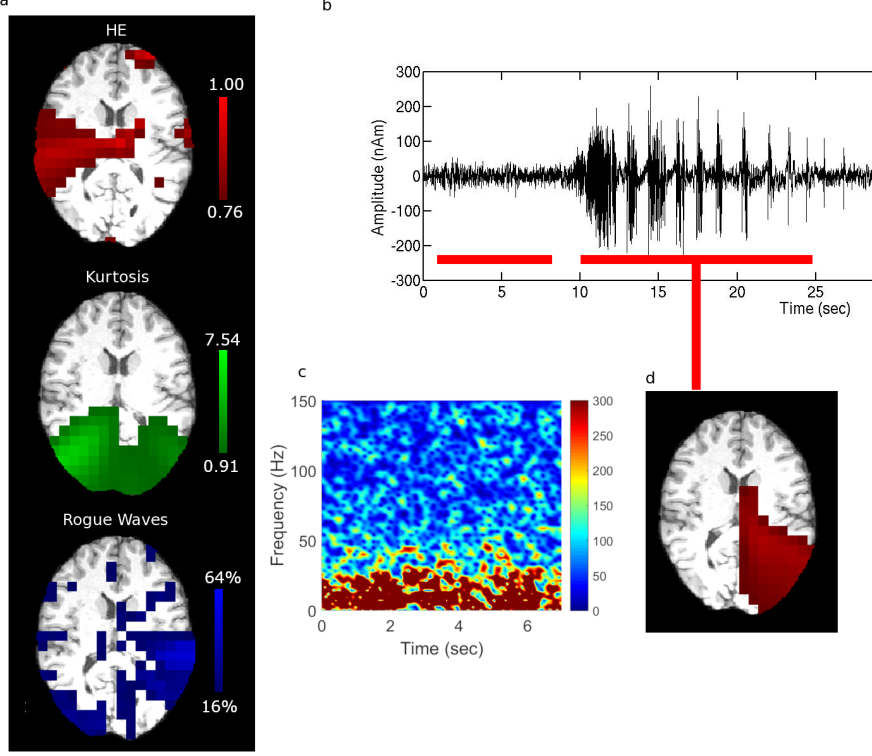

573

574

Figure 4

Data for Patient 3, who had a seizure during the recording. Figure 4a shows the same slice from the thresholded volumetric images for each of $\mathrm{HE}$, kurtosis, and percent rogue waves, for the period

577 before the seizure. Figure $4 \mathrm{~b}$ shows the timeseries from the source with peak HE values in the frontal lobe, for the entire recording, with the periods before, during and after the seizure identified with red bars. Figure $4 \mathrm{c}$ is a spectrogram of the pre-seizure activity. Figure $4 \mathrm{~d}$ and $4 \mathrm{e}$ show the same 580 581

582 slice from the volumetric $\mathrm{HE}$ images during (4d) and after (4e) the seizure. 
Table 1. A summary of the 3 patient case-studies.

584

\begin{tabular}{|l|l|l|l|l|l|l|}
\hline Patient & Sex & $\begin{array}{l}\text { Age at } \\
\text { MEG } \\
\text { recording }\end{array}$ & Diagnosis & $\begin{array}{l}\text { Epileptogenic } \\
\text { zone and how } \\
\text { determined }\end{array}$ & $\begin{array}{l}\text { Surgery and } \\
\text { outcome }\end{array}$ & $\begin{array}{l}\text { Clinically } \\
\text { ascertained } \\
\text { epileptogenic } \\
\text { zone location }\end{array}$ \\
\hline 1 & Female & 16 years & $\begin{array}{l}\text { Focal } \\
\text { epilepsy }\end{array}$ & $\begin{array}{l}\text { Corticography } \\
\text { identified a left } \\
\text { temporal } \\
\text { epileptogenic } \\
\text { zone }\end{array}$ & $\begin{array}{l}\text { Surgical resection } \\
\text { of the left } \\
\text { temporal } \\
\text { epileptogenic zone } \\
\text { resulted in } \\
\text { eradication of } \\
\text { seizures }\end{array}$ & $\begin{array}{l}\text { Source in left } \\
\text { temporal lobe }\end{array}$ \\
\hline 2 & Male & 8 years & $\begin{array}{l}\text { Right parietal } \\
\text { Glioma }\end{array}$ & $\begin{array}{l}\text { Corticography } \\
\text { showed interictal } \\
\text { discharges in the } \\
\text { frontal as well as } \\
\text { parietal lobe. }\end{array}$ & $\begin{array}{l}\text { Right parietal } \\
\text { resection resulted } \\
\text { in a reduction in } \\
\text { seizure frequency } \\
\text { but not eradication } \\
\text { of seizures } \\
\text { suggesting that the } \\
\text { frontal source was } \\
\text { also epileptogenic }\end{array}$ & $\begin{array}{l}\text { Sources in } \\
\text { right parietal } \\
\text { and left } \\
\text { frontal lobes }\end{array}$ \\
\hline 3 & Female & 32 years & $\begin{array}{l}\text { Focal } \\
\text { epilepsy }\end{array}$ & $\begin{array}{l}\text { Corticography } \\
\text { identified a right } \\
\text { frontal source. }\end{array}$ & $\begin{array}{l}\text { Right frontal } \\
\text { source confirmed } \\
\text { post surgically to } \\
\text { be the } \\
\text { epileptogenic } \\
\text { source }\end{array}$ & $\begin{array}{l}\text { Source in } \\
\text { right frontal } \\
\text { lobe }\end{array}$ \\
\hline
\end{tabular}


587

588

589

590

591

592

593

594

595

596

597

598

599

600

Table 2. Percent overlap between the thresholded volumetric images for the kurtosis, rogue waves, and HE measures, and the mean and range of percent overlap values for the 6 possible comparisons between independent images across the three particpants. Data for Patient 3 are based on the preseizure activity only; see Figure 4 for details.

\begin{tabular}{|l|l|l|l|l|}
\hline Concordance & Patient 1 & Patient 2 & Patient 3 & $\begin{array}{l}\text { Reference: } \\
\text { concordance } \\
\text { between patients } \\
29 \%(15 \%-39 \%)\end{array}$ \\
\hline HE - Kurtosis & $42 \%$ & $33 \%$ & $16 \%$ & $29 \%(13 \%-43 \%)$ \\
\hline $\begin{array}{l}\text { Rogue waves - } \\
\text { Kurtosis }\end{array}$ & $77 \%$ & $19 \%$ & $48 \%$ & $24 \%(10 \%-32 \%)$ \\
\hline HE- Rogue waves & $44 \%$ & $36 \%$ & $30 \%$ & 27 \\
\hline
\end{tabular}

Table 3.

PSI values for Patient 1 in 1-150 Hz frequency band, for each pair of locations identified from peaks in the cortical $\mathrm{H}$ maps and shown Figure 1b. Significant PSI values, i.e. those exceeding 1.96 standard deviations, are in bold, with positive values and sources representing a 'driving' relationship further highlighted in yellow.

\begin{tabular}{|l|l|l|l|l|l|}
\hline & L. Temporal & L. Frontal & R. Occipital & R. Parietal & Control \\
\hline L. Temporal & 0 & 3.91 & $\mathbf{2 . 3 1}$ & $\mathbf{3 . 7 2}$ & $\mathbf{2 . 3 1}$ \\
\hline L. Frontal & -3.91 & 0 & -1.86 & -1.42 & -1.69 \\
\hline R. Occipital & $-\mathbf{2 . 3 1}$ & 1.86 & 0 & 1.21 & -0.19 \\
\hline R. Parietal & $-\mathbf{3 . 7 2}$ & 1.41 & -1.21 & 0 & -0.41 \\
\hline Control & -2.31 & 1.69 & 0.19 & 0.41 & 0 \\
\hline
\end{tabular}


601

602

603

604

605

606

607

608
Table 4.

PSI values for Patient 2 in 1-150 $\mathrm{Hz}$ frequency band, for each pair of locations identified from peaks in the cortical $\mathrm{H}$ maps and shown Figure $1 \mathrm{k}$. Significant PSI values, i.e. those exceeding 1.96 standard deviations, are in bold, with positive values and sources representing a 'driving' relationship further highlighted in yellow.

\begin{tabular}{|l|l|l|l|l|l|}
\hline & L. Frontal & R. Parietal & L. VL Frontal & R. Occipital & Control \\
\hline L. Frontal & 0 & -5.03 & -2.96 & 0.35 & 0.96 \\
\hline R. Parietal & $\mathbf{5 . 0 3}$ & 0 & 0.54 & $\mathbf{5 . 2 3}$ & $\mathbf{7 . 1 8}$ \\
\hline L. Ventrolateral Frontal & $\mathbf{2 . 9 6}$ & -0.54 & 0 & $\mathbf{3 . 5 0}$ & $\mathbf{4 . 1 6}$ \\
\hline R. Occipital & -0.35 & $-\mathbf{5 . 2 3}$ & & 0 & 1.73 \\
\hline Control & -0.96 & $-\mathbf{7 . 1 8}$ & -4.16 & -1.73 & 0 \\
\hline
\end{tabular}


609

610

611

612

613

614

615

616

\section{Table 5}

PSI values for Patient 3 in the 4-30 Hz frequency band, for each pair of locations identified from peaks in the pre-seizure cortical $\mathrm{H}$ maps and shown Figure 2c. Significant PSI values, i.e. those exceeding 1.96 standard deviations, are in bold, with positive values and sources representing a 'driving' relationship further highlighted in yellow.

\begin{tabular}{|l|l|l|l|}
\hline & R. Frontal & R. Parietal & L. Temporal \\
\hline R. Frontal & 0 & $\mathbf{2 . 6 7}$ & $\mathbf{3 . 5 7}$ \\
\hline R. Parietal & $\mathbf{- 2 . 6 7}$ & 0 & 1.28 \\
\hline L. Temporal & $\mathbf{- 3 . 5 7}$ & -1.28 & 0 \\
\hline
\end{tabular}


4

5

6

8

\section{Supporting Information: Movie Legends}

\section{SI Movie 1}

619 Spectrogram animation of the data for Patient 1 from the whole recording period, reconstructed

620 from the source in the left temporal lobe in Figure $1 \mathrm{~b}$ (the same data for which the timeseries is

621 shown in Fig 1d). This source had the highest $\mathrm{H}$ value and was identified as the driving epileptogenic

622 source.

623

624

SI Movie 2

625 Spectrogram animation of the data for Patient 2, for the whole recording period, reconstructed from 626 the source in the left frontal lobe that was identified as one of the driving epileptogenic sources.

627 Timeseries for these data are also shown in Fig $1 \mathrm{~m}$.

628

629

630

631

632

633

634 635

636

\section{SI Movie 3}

Spectrogram animation of the data for Patient 3, reconstructed from the beamformer weights for the pre-seizure period in the right frontal epileptogenic source, but for the whole timeseries so changes during and after the seizure can also be seen.

\section{3}

(3)

5
7
8
9
0
1
2
3
4
4
47
4
49
5
51
53
5
5
5

